\title{
MAC Protocol for Supporting Multiple Roaming Users in Multi-Cell UWB Localization Networks
}

\author{
Nicola Macoir, Matteo Ridolfi, Jen Rossey, Ingrid Moerman, and Eli De Poorter \\ Ghent University - imec, IDLab, Department of Information Technology
}

\begin{abstract}
Indoor positioning systems (IPS) aim to track objects, people or assets with the highest possible accuracy. In literature, it has been shown that among radio-frequency based technologies, ultra-wideband (UWB) is capable of providing cmlevel accuracy. However, this technology is not yet mature when it comes to large-area coverage and multi-user support. To remedy this, this paper proposes a TDMA protocol for a large scale localization network with numerous simultaneously active users. To this end, a full system was designed that handles the scheduling of the transmissions, the synchronization of the fixed nodes and the roaming of the mobile nodes. Moreover, the performance of the system has been analyzed by simulations using OMNeT++ and INET framework. The simulation was improved with real life experiments and has been used to evaluate full TDMA/TDoA approach. Our solution improves the scalability to $88.3 \%$ effective spectrum usage, compared to only $18.6 \%$ when using ALOHA, while mobile nodes are able to roam successfully in $90 \%$ of the handovers.
\end{abstract}

Keywords-UWB, Indoor Positioning Systems, MAC Protocols, OMNeT++

\section{INTRODUCTION}

D etermining the position of mobile assets using Radio Frequency (RF) based solutions can be challenging. There exist several technologies such as Bluetooth Low Energy (BLE), ZigBee, WiFi and UWB to handle this task. Of these, UWB seems to be the most promising standard when it comes to accuracy, offering $\mathrm{cm}$-level accuracy.

Although UWB localization has been studied for many years, the focus of UWB research has mainly been the need for better positioning accuracy. Example recent scientific publications analyze how UWB is capable of providing cm-level accuracy [1], even mm under certain circumstances [2]. These high positioning accuracy results, together with the recent availability of affordable UWB chips on the market [3], make ultrawideband the perfect choice for indoor localization systems. Nonetheless, these scientific papers investigate only open, smallscale controlled environments such as a single office room. As such, they do not analyze or improve other localization aspects such as large-area coverage, tag roaming and multi-user interference, which are crucial to scale towards realistic indoor deployments.

In this paper, we focus on these less studied aspects, evaluating the feasibility to scale up the use of UWB in different applications requiring large-scale coverage and multiuser support. These forms of scalability are closely related to MAC protocol design since the system must provide fair access to as many users as possible while optimizing its resources and available spectrum. Unfortunately, due to the low radiation power density and the sparse and transient nature of impulse UWB radio, conventional clear channel assessment (CCA) techniques which detect the energy of carrier waveforms cannot easily be applied to UWB technologies [4]. As a consequence, most UWB systems currently use slotted ALOHA to fragment the time in slots and let the nodes transmit at the start of a random chosen slot. However, when the number of users increases, slotted ALOHA is known to scale poorly [5, 6], and as such dense multi-user environments will result in a significant amount of collisions and degraded performance.

Therefore, our starting point is a multi-cell TDMA protocol, which divides the time in slots to be assigned to different users in order to avoid collisions. Moreover, when considering a large area with multiple fixed nodes, the mobility of the tags (nodes to be tracked) plays an important role as they move unpredictably from one part of the network, i.e. roaming between different cells. To this end, we designed a multi-cell MAC protocol and management algorithms to cope with challenges such as multi-cell slot allocations, cell handovers and resource re-usage.

\section{RELATED WORK}

This section is divided into two parts. First TDMA scheduling algorithms used in multi-hop communication networks are discussed. Afterwards, we discuss recent research on the accuracy and scalability of UWB localization networks.

\section{A. Multi-hop TDMA}

In wireless networks, the most popular medium access control scheme is the IEEE 802.11 Distributed Coordination Function (IEEE 802.11 DCF)[7]. However, in multi-hop environments this scheme suffers from the fairness problem, which is caused by the existence of hidden terminal and exacerbated by the adopted binary exponential backoff algorithm to resolve contention [8]. Furthermore, the IEEE 802.11 DCF has numerous disadvantages such as high overhead, increased access delay, high jitter and limited QoS capabilities. TDMA seems to be a more promising solution, since it can overcome these issues. However, a solution for the NP-complete Broadcast Scheduling Problem (BSP) [9] is needed for using TDMA in a wireless multihop environment.

End-to-end link scheduling TDMA multi-hop research papers focus on the design of link scheduling algorithms for finding optimal slot schedules to support end-to-end connectivity between mobile stations and a gateway [10]. This research is not applicable to TDoA-based indoor localization since in our network, nodes will always broadcast messages to anchor nodes and no multi-hop point-to-point links are required for localization.

Instead, our work is more closely related to broadcast scheduling algorithms, which aim to find optimal slot schedules that allow several mobile nodes to broadcast their transmission without interfering each other. Several TDMA broadcast scheduling algorithms can be found in scientific literature. These algorithms use heuristic approaches such as "greedy" algorithms [9], mean field annealing [11] or genetic algorithms [12] in order to solve the Broadcast Scheduling Problem (BSP). These algorithms aim to derive the optimum slot allocation and superframe length to maximize the network throughput. However, maximizing the overall network throughput can result in an unfair allocation of resources to users [13] and 
these approaches do not suit the specific needs of localization algorithms that require specific update rates.

Finally, in [14], an algorithm for collision-free time slot reuse is defined which allows nodes to be self-configuring and discover which time slots can be used without interfering with other nodes in their second order neighborhood (thereby causing collisions). In contrast, in our use case the mobile nodes have to broadcast messages to more then one receiving anchor node. Also, the distributed nature of the algorithm is not necessary since we can utilize the localization context, and use the obtained position to assign slots efficiently to the mobile nodes. This is the case of [15], wherein the authors uses Spatial-TDMA (STDMA) to track and monitor mobile nodes in the network. They design a topology-transparent broadcast scheduling protocol that ensures collision-free transmission within a 2-hop neighbourhood. Mobility of stations is taken into account. However, the approach is not technology specific and therefore not optimized for localization networks, whose performance may be affected by MAC layer delays.

In summary, our multi-cell TDMA MAC algorithms do not aim to schedule end-to-end traffic, but focus on localization specific traffic. Furthermore it is possible for mobile nodes to request multiple slots (e.g. when they prefer a high localization update frequency). Fairness is achieved by scheduling every client in the order that they request a slot (FIFO) and by giving priority to those users that were not able to obtain a slot in the previous superframe.

\section{B. UWB Localization}

As discussed before, recent UWB work focuses mostly on accuracy-improvements for indoor localization, rather than scalability and large-area coverage. This section gives an overview of UWB localization research focusing on these last aspects.

In [16], the authors study the navigation of a robot in a larger size area than what is usual done in other works. They implement a 3-cell UWB system and still achieve good accuracy result, i.e. average errors around $15 \mathrm{~cm}$. However, the problem is tackled without considering MAC features since only one robot is using the network. Instead, their work focuses on boundary detection and robot path prediction. The central server is in charge of these two tasks and the results show that seamless handover is possible. In contrast, our work supports scalable roaming of multiple tags using a TDMA MAC protocol.

In [17] the authors optimize the scheduling of the network resources with localization constraints in UWB networks. The work is split into several local link selection sub-problems and one centralized scheduling problem. This is done to overcome the fact that in large scale networks the optimal solution they analyze, is not applicable since its complexity grows very fast. However, their work considers two way ranging (TWTOA) as localization technique where every tag transmit to each individual anchor node. In our work, Time Difference of Arrival (TDoA) is used as localization technique, since it allows tags to broadcast their transmissions. Furthermore, the solution lacks large-scale deployments with more than 4 cells and does not include simulations with more realistic parameters, which we tackle in our work.

\section{SyStem ARCHITECTURE AND MODEL}

This paper presents an UWB indoor localization system using Time Difference of Arrival (TDoA) localization approach

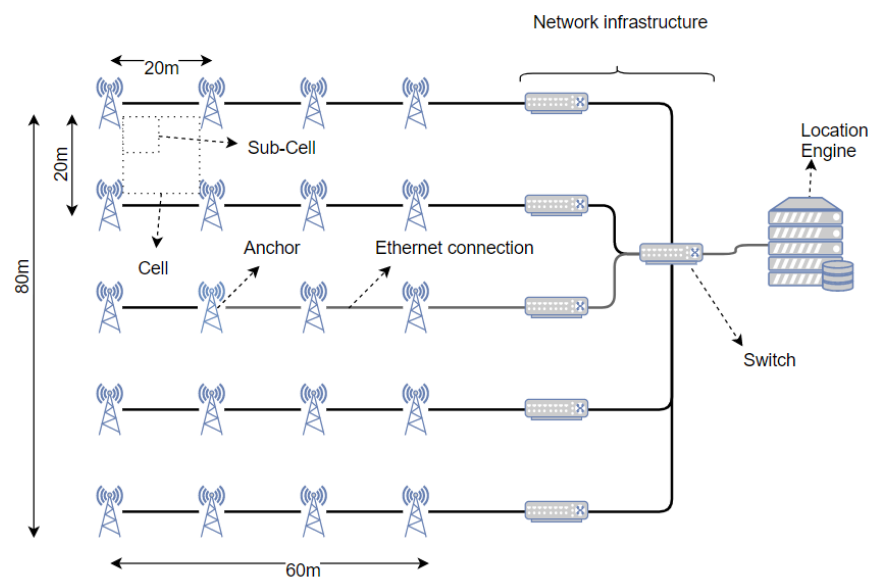

Fig. 1: Centralized system architecture with multiple fixed anchors

that ensures fair and collision-free access to the network through a TDMA protocol. These design choices impact the realization of the network, requiring specific and optimized solutions for managing, coordinating and synchronizing multiple anchor nodes. Therefore, specific solution related to network planning and MAC protocol design are presented in the next three paragraphs.

\section{A. Network architecture}

We introduce the concept of cell as spatial areas of the network served by multiple anchor nodes. The purpose is to subdivide the network in cells and to identify the best slot allocation per cell, which would guarantee the maximum number of supported users (tags) while using the minimum amount of resources (slots). In Figure 1, an example is shown. The example network is composed of a $5 \times 4$ grid resulting in 20 anchor nodes. Anchor nodes are $20 \mathrm{~m}$ apart from each other, encompassing an overall area of $80 \times 60 \mathrm{~m}$ that is covered by 12 cells. The overall $480 \mathrm{~m}^{2}$ area is subdivided in 12 cells and each cell is further composed of 4 sub-cell (see Section IV-A).

Each anchor row is connected to a switch, in turn linked to a higher level switch directly communicating with the central server or the Location Engine (LE). The location engine is a server or powerful computing device who is mainly responsible for processing the TDoA ranging measurements and calculating the position of each tag. Beside that, it calculates the scheduling and it predicts the roaming for the mobile tags. In this example, the infrastructure nodes are connected via Ethernet but our solution also supports other implementations, e.g. in [18] the fixed nodes are connected via Wi-Fi in a mesh network.

\section{B. TDMA Protocol and Clock Synchronization}

Tags and anchors communicate with each other using the Impulse Radio (IR) UWB PHY described in the IEEE 802.15.4a standard [19], which allows precision ranging, and is very robust even at low transmit powers. The MAC protocol described in the IEEE 802.15.4-2011 standard [20] includes several concepts such as a dynamic superframe structure, the association process and guaranteed timeslots (GTS) that are also utilized in our MAC protocol. A standard TDMA approach divides the time in superframes and each superframe is typically composed of other portions such as synchronization (scheduling) period, contention access period (CAP), and contention free period (CFP) as illustrated in Figure 2. Beacons which contain the slot 
schedule for the mobile nodes are sent periodically by some designated anchor nodes. After the reception of the beacons, the Contention Access Period (CAP) begins which can be used by the tags to send slot requests using slotted ALOHA. Eventually, during the contention free period, dedicated time slots are provided for those tags that were allocated one or more slots. Because the anchors need to communicate with the server at some point, a small time slot $t_{s r v}$ is reserved. Each of these phases will be discussed in more detail below.

1) Synchronization phase: Accurate time synchronization is a crucial aspect in TDoA-based localization systems. For example, a clock difference of $1 \mathrm{~ns}$ between two anchors would result in an accuracy error of $30 \mathrm{~cm}$. One way to synchronize the clock of the anchor nodes of a cell is to use an Ethernet based time synchronization protocol. However, most commercially available standardized synchronization protocols such as the Network Time Protocol (NTP) and the Precision Time Protocol (PTP) only reach synchronization accuracies between 10 and 100 microseconds (software implementations) or synchronization accuracies of 30 nanoseconds (specialized hardware) [21], both of which are insufficiently accurate for TDoA ranging. In [22], enhanced methods were proposed for up to now unmatched timestamping accuracy in Ethernet-based synchronization protocols. The methods from this last paper reach sub-nanosecond accuracy, which is proven in theory and practice. However, the proposed solution requires custom, not off-the-shelf hardware and is not proven to be scalable for large networks with longer distances between the server and the anchor nodes.

For this reason, we use the Ethernet connections only to exchange data messages between anchor nodes and server. Instead, we opt to use the UWB radio for both localization and time synchronization. Assuming a sufficiently fast clock, this results in a time synchronization accuracy of around 1 nanosecond.

This synchronization mechanism is easy to apply to one cell, e.g. for one-hop synchronization. However, in order to synchronize multiple anchors in multi-hop deployments, we need to propagate multiple synchronization messages. The synchronization process is repeated each superframe and starts with the server sending an Ethernet message to one central anchor node to start the initialization process. This anchor node transmits an UWB message to all anchor nodes within range (one-hop anchor nodes). Afterwards, the one-hop anchor nodes in turn transmit UWB messages to synchronize the two-hop distance anchor nodes. Since a single UWB synchronization message reaches multiple anchor nodes, not all anchor nodes should further distribute the synchronization messages. To determine which anchor nodes should further propagate the UWB synchronization messages, the Trickle algorithm is used [23]. A node using the Trickle dissemination algorithm sends out its data in a probabilistic way using slotted ALOHA, unless it has recently received a message which contents are identical. The use of Trickle ensures that not all anchor nodes transmit synchronization beacons, thereby reducing the synchronization phase duration $T_{\text {sync }}$, but still result in every anchor node receiving multiple synchronization messages which can be averaged out to improve synchronization accuracy.

To accommodate the sequential multi-hop UWB synchronization process using Trickle, the Sync period in Figure 2 is sufficiently large for transmitting multiple sequential UWB syn-

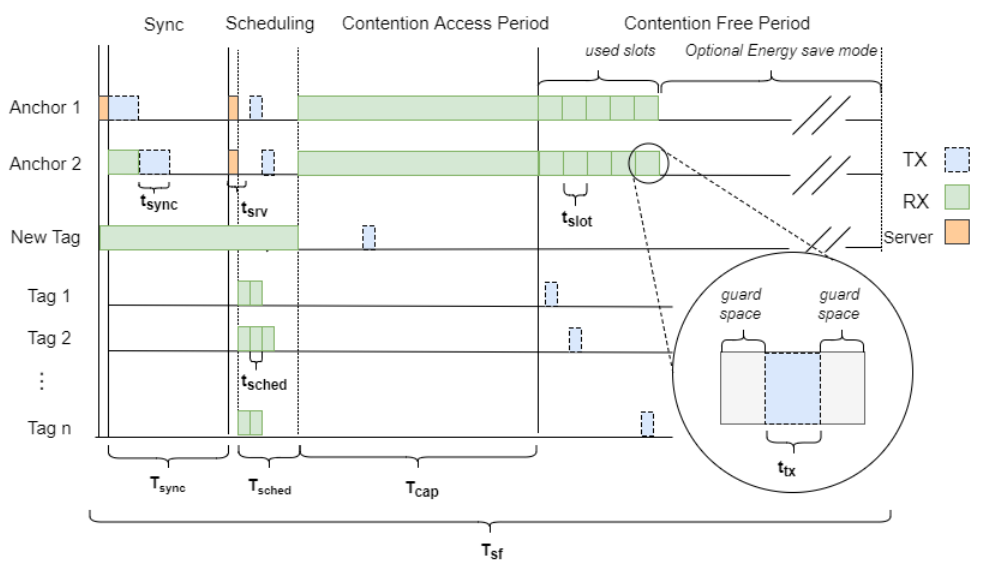

Fig. 2: Structure of the TDoA-TDMA UWB MAC Protocol, with indication of timing parameters and message exchanges between anchors and tags.

chronization messages of $t_{\text {sync }}$ duration between the different cells.

2) Scheduling phase: During this phase, anchor nodes use broadcast messages to inform the mobile tags about the slots that are allocated. The slot assignment procedure is discussed in Section IV-B). Multiple anchors can simultaneously send messages at the same time provided that they are separated by at least twice the distance of their signal range. To keep the number of transmitting anchors minimal, rather than distributing this information from every anchor node, every cell has a single master anchor who is responsible for distributing the scheduling information for that cell. Once a mobile tag receives an allocated slot, the radio of the tag is turned off until the beginning of the slot.

3) Contention period: Tags that did not receive a timeslot (e.g. tags that recently joined the network), can retrieve the starting time of the next Contention Access Period in the scheduling beacon. During this period, new tags can send a slot request for the next superframe(s) using ALOHA. When collisions occur in this period, the tag will try again in the next superframe. A slot request is successful when it is received by minimum three anchor nodes, so that the position of the slot requester can be calculated.

4) Contention free period: After the CAP period, the contention free period starts, during which every tag that was allocated a slot, can use this slot to transmit it's TDoA ranging packet to the network without interfering with other nodes. A mobile node can set their radio's into sleep mode during the other slots and for the remaining period of the superframe. In case only few mobile nodes are present in the cell, also the anchor nodes can enter an energy saving mode for the duration where no slots are allocated.

\section{Mobility Management}

In this section, we introduce the roaming and scheduling algorithms, which are used by the server to calculate the slots for the next superframe. Simulation results of our algorithm are shown in the next section.

\section{A. Division into cells and sub-cells}

Each network cell is further sub-partitioned into 4 sub-cells, which are used during the roaming and scheduling procedures to calculate sets of non-interfering cells (see Figure 3). Most of the anchors (except the corner anchors) function as anchor node 


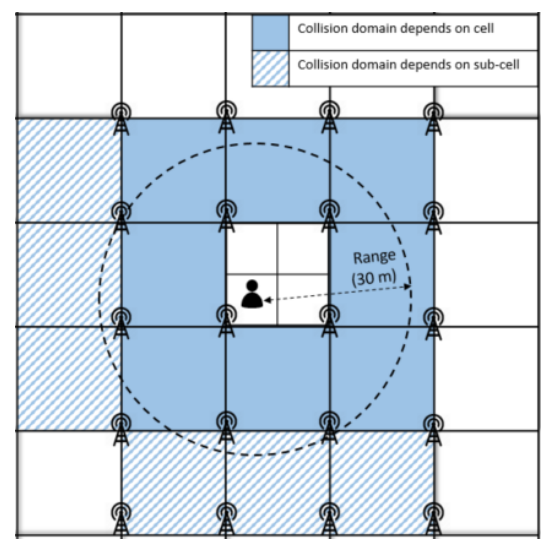

Fig. 3: Calculation of the collision set of mobile tags. Neighboring cells are always included in the collision set. A number of additional two-hop distance cells are also included, depending on the transmission range and the sub-cell the mobile tag is located in.

for more then one cell, i.e. they receive ranging packets from mobile tags from all surrounding cells. As such, assuming that only a single radio frequency is used, two tags that are located in cells next to each other can only transmit without interfering each other if they have obtained a different timeslot. Hence, to optimally make use of the spectrum, reusage of slots can be achieved on condition that tags are not two-hop neighbors. Because the transmission range from a tag should be larger then the cell width to be able to reliable range with at least 4 anchor nodes, we have to make sure all tags that are using the same timeslot are not in each other collision domain.

The collision domain associated to a particular cell contains all it's one-hop neighbor cells. However, as seen in Figure 3, depending if the tag is located north/south or east/west in the cell, the transmission range can even reach the outer anchors from neighbor cells, thereby interfering even with cells that are at two-hop distance. At initialization a set of cells, defined as collision set, is set for each pair (Cell, sub-cell). When assigning a timeslot to multiple clients, the system verifies these devices are not in each other collision set.

\section{B. Slot Assignment}

Upon the reception of a new slot request, the server first checks whether there are still free slots. If that is the case, then the server simply assigns one of the free slots to the tag. The number of available time slots can be calculated by using (1):

$$
n_{\text {slots }}=\frac{T_{s f}-T_{\text {cap }}-2 t_{\text {srv }}-T_{\text {sync }}-T_{\text {sched }}}{t_{\text {slot }}}
$$

where $T_{\text {sf }}, T_{\text {cap }}, T_{\text {sync }}, T_{\text {sched }}, t_{\text {srv }}$, and $t_{\text {slot }}$ are the lengths of superframe, CAP period, synchronization period, scheduling period and the slot lengths of a server message and ranging message, respectively.

When the number of users increases and all slots have been assigned, the server aims to allocate already assigned slots also to newly joining mobile tags ("slot reuse"), while considering the presence of interfering cells. As discussed in Section IV-A, for each cell and sub-cell in the network there is a subset of interfering cells. The server keeps a list of all the possible inferring cells for each tag based on which it will determine if one slot can be assigned to two or more different tags. More precisely, the server assigns a slot to a tag using Algorithm 1, which ensures that there are no situations wherein one slot is shared by tags that are in the same collision domain.

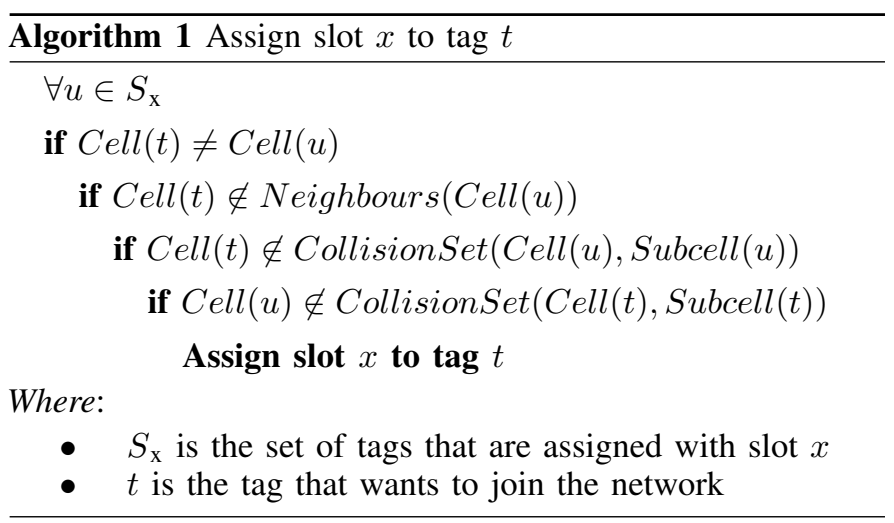

In case a tag can not be assigned a slot, either due to system limits (overcrowded network) or due non-optimal slot assignment, the tag request is queued and a flag is set to inform that during the next iteration (next superframe) the server will re-calculate the schedule, starting by allocating slots for the tags that were unsuccessful last superframe.

Finally, slots will also become available when tags have moved to a new cell. Every tag that is registered on the LE has a time to live (TTL) value assigned. In every superframe, the TTL is decreased for all tags that could not successful be localized. This way, the system can detect when a tag has left the network or when it becomes unreachable, and free the allocated slots.

\section{Handover Detection}

The handover or roaming process refers to the mechanism with which the server recognizes when a tag moved from one cell to another, i.e it is now transmitting from a new position and its messages are received by a different set of anchors. Predicting when such a handover occurs is important for calculating the two-hop collision free slot schedules.

In localization systems, the server is aided in the handover process due to the availability of estimated positions of the tags. To this end, standard multilateration algorithms can be applied: in TDoA schemes, the coordinates of the mobile nodes are estimated by comparing the difference of the arrival times among all the anchors that have received the message from the tag. Each time the current position is computed, the server will also predict the position of the tag in the next superframe using the estimated acceleration $a$, the velocity $v$ and the direction $\theta$. If the mobile tags are equipped with additional sensors, i.e. accelerometer, gyroscope and magnetometer, these sensors can be used to estimate the above variables. If not, the server estimates the velocity and acceleration based on previous location estimates. We can predict the location of the tag in the following $n$ superframes by using (2)-(3).

$$
\begin{aligned}
& x=x_{0}+\left(v \cdot T_{s f}+\frac{1}{2} \cdot a \cdot T_{s f}^{2}\right) \cdot n \cdot \cos \theta \\
& y=y_{0}+\left(v \cdot T_{s f}+\frac{1}{2} \cdot a \cdot T_{s f}^{2}\right) \cdot n \cdot \sin \theta
\end{aligned}
$$

If the expected location is situated in a new (sub-)cell, roaming is predicted and the validity of current slot must 


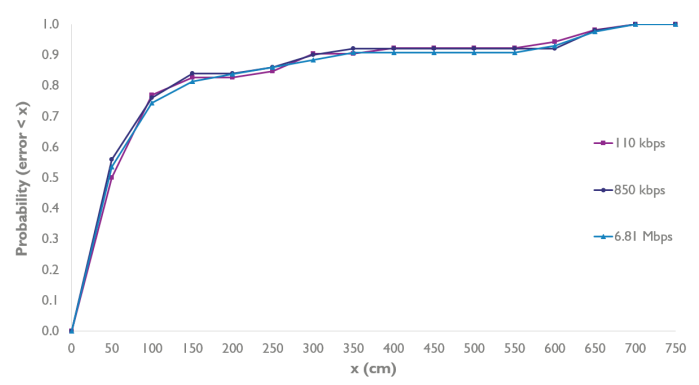

Fig. 4: Probabilistic UWB positioning error model based on experiments in the WiLab.t wireless industrial testbed facility using the DW1000 UWB chip.

be checked using the procedure outlined in Section IV-B. In case a tag roamed without the server predicting it, the tag might transmit using a slot that is already utilized by another mobile node. In this case, most likely a collision will occur and the tag will get notified in the next scheduling message. After certain failed attempts, the tag will finally send a new slot request.

\section{Simulation Setup}

To evaluate our design, we used the popular OMNeT++ [24] simulator to create a customized network and modules based on the INET framework. OMNeT++ allows simulation of all protocol layers of both the UWB wireless communication as well as the wired ethernet links. Some assumptions have been made: the tags have all the same update frequency $(1 \mathrm{~Hz})$, identical communication range $(30 \mathrm{~m})$ and the propagation is ideal, i.e. the cells are represented by square portions of the network. Since the OMNeT simulator lacks support for clockdrift, it generates positioning estimates that are too optimistic. Therefore, experiments were first performed in the WiLab.t wireless testbed facility [25], a challenging industrial testbed with several metal obstacles, to obtain typical UWB position estimate error models (see Figure 4). This probabilistic error model is used by the simulator during each position estimate of the simulator to improve the realism of the simulator.

\section{A. TDMA Parameters}

Before collecting results in our simulation, realistic parameters for the TDMA protocol and the message duration settings need to be chosen. The simulation uses the parameters of Table I. With reference to [26], we assume that a clock synchronization rate of around $1 \mathrm{~Hz}$ is a good compromise in terms of synchronization overhead and positioning error. For this reason, the superframe duration $T_{s f}$ is defined as 1 second.

Most UWB radios support multiple operational modes with different preamble lengths, payload sizes, etc. Different operational modes can be found in the Decawave DW1000 datasheet [3]. We will use UWB Channel 2; which has a center frequency of $3993.6 \mathrm{MHz}$ and a bandwidth of $499.2 \mathrm{MHz}$. In Table II, we list the most interesting radio configurations for our protocol, including their average energy consumption for transmission and reception. (i) For acquiring high precision when synchronizing, operational mode 9 with a long preamble and high Pulse Repetition Function (PRF) is used to have a better first path time of arrival information. However, this comes at the price of a longer message airtime and increased energy consumption consumption. (ii) Next, for distributing the scheduling messages, a high bitrate and longer message size is required, which makes operational mode 6 a good choice. (iii) Finally, for the ranging messages from the mobile
TABLE I: SYSTEM VARIABLES

\begin{tabular}{|c|c|c|}
\hline Variable & Meaning & Value \\
\hline$n_{\text {tags }}$ & Number of tags & {$[5,10]$} \\
\hline$n_{\text {anchors }}$ & Number of anchors & 20 \\
\hline$n_{\text {slots }}$ & Number of ranging slots & 5 \\
\hline$n_{\text {synch }}$ & Number of synchronization slots & 4 \\
\hline$n_{\text {sched }}$ & Number of scheduling slots & 5 \\
\hline$v_{\mathrm{t}}$ & Tag speed & $1-3 \mathrm{~m} / \mathrm{s}$ \\
\hline$T_{\text {sf }}$ & Superframe length & $1 \mathrm{~s}$ \\
\hline$T_{\text {sync }}$ & Synchronization phase length & $10 \mathrm{~ms}$ \\
\hline$T_{\text {sched }}$ & Scheduling phase length & $5 \mathrm{~ms}$ \\
\hline$T_{\text {cap }}$ & CAP length & $100 \mathrm{~ms}$ \\
\hline$t_{\text {srv }}$ & Server message slot length & $1 \mathrm{~ms}$ \\
\hline$t_{\text {sync }}$ & Synchronization slot length & $2.5 \mathrm{~ms}$ \\
\hline$t_{\text {sched }}$ & Scheduling slot length & $1 \mathrm{~ms}$ \\
\hline$t_{\text {slot }}$ & Localization Slot length & $0.5 \mathrm{~ms}$ \\
\hline
\end{tabular}

nodes, operational mode 10 is chosen. This includes short message duration and a high PRF, which will result in accurate positioning, but again comes with a price of additional power consumption. A short preamble (128 symbols) is chosen, because when using a high datarate, the operating range is short and there is no point in sending a very long preamble as it wastes time and power for no added range advantage. The most interesting operational modes for ranging is thus mode 10 .

\section{B. Mobility model}

To test the roaming algorithm, two mobility models were implemented. The first model has linear behavior, or in other words, the mobile node moves trough the space in a constant direction (uniform initiated) until it reaches the borders, after which the mobile node will be reflected in the other direction. Since this behaviour is very predictable, a more natural mobility model is also included where nodes are walking randomly trough the space (e.g. users in a supermarket mostly are grabbing products from one shelve and move to another). The second mobility model calculates a random target position for the mobile node. Depending to the update interval and the velocity it calculates the number of steps to reach the destination and the step-size. Every update interval the module calculates the new position on its way to the target position and updates the display. Once the target position is reached the module calculates a new target position.

\section{RESULTS}

Finally, the performance of the proposed system is evaluated using both theoretical and simulation results. The different metrics were evaluated by executing for each experiment 10 simulations, each with different random seed, resulting in different situations. 
TABLE II: CHOICE OF OPERATIONAL MODE FOR EACH TDMA SUPERFRAME PHASE.

\begin{tabular}{|c|c|c|c|c|c|c|c|c|}
\hline Mode & Data Rate & PRF & Preamble & Data length & Packet Duration & Avg TX Current & Avg RX Current & Phase \\
\hline 6 & $6.8 \mathrm{Mbps}$ & $16 \mathrm{MHz}$ & 128 Symbols & 127 bytes & $312 \mu \mathrm{s}$ & $56 \mathrm{~mA}$ & $113 \mathrm{~mA}$ & Scheduling \\
\hline 9 & $110 \mathrm{kbps}$ & $64 \mathrm{MHz}$ & 1024 Symbols & 12 Bytes & $2469 \mu \mathrm{s}$ & $61 \mathrm{~mA}$ & $90 \mathrm{~mA}$ & Synchronization \\
\hline 10 & $6.8 \mathrm{Mbps}$ & $64 \mathrm{MHz}$ & 128 Symbols & 12 bytes & $179 \mu \mathrm{s}$ & $79 \mathrm{~mA}$ & $112 \mathrm{~mA}$ & Ranging \\
\hline
\end{tabular}

\section{A. Scalability}

First, we compare the maximum number of users of our TDMA design with traditional ALOHA based UWB solutions. Based on existing scientific literature [5], the recommended maximum channel utilization when using pure ALOHA equals 0.186 which means that only $18.6 \%$ of the time is used for communications. In this case, $97 \%$ of transmissions are likely to succeed without collisions. Further increasing channel occupancy will result in a rapid increase of the number of collisions. In [6], it is proven that the capacity can be doubled $(36 \%)$ for slotted ALOHA. As such, using the slot lengths as discussed in Section V-A, 720 slots are available in every superframe when using slotted ALOHA.

In comparison, for our TDMA configuration using the same slot sizes, up to $88.3 \%$ of the time can be used for communications among nodes. As a result, up to 1766 timeslots are available per cell. Furthermore, in contrast to slotted ALOHA, slots can be shared among tags if they are located far enough from each other. We can conclude that in comparison to slotted ALOHA, the TDMA capacity of the network is more than doubled for a single cell, and is even further improved when considering slot reuse among cells (see Section VI-C).

\section{B. Roaming success rate}

To evaluate the roaming success rate multiple experiments were done with velocities varying from 1 to $3 \mathrm{~m} / \mathrm{s}$, using the different mobility models described in Section V-B. In every experiment, we let all nodes move at the same velocity and using the same mobility model for 60 seconds. The roaming system verifies based on the predicted position of the next localization if the tag will move from one cell to another. The prediction can be wrong because of several factors, such as unpredictable movement changes or incorrect position estimates caused by the probabilistic position error model described in Section V.

In Figure 5, the roaming success rate is shown in function for the different velocities using both the linear mobility model and the random waypoint mobility model. The roaming success rates vary from $100 \%-93 \%$ at normal walking speed $(1 \mathrm{~m} / \mathrm{s})$ to $98 \%-89 \%$ at running speed $(3 \mathrm{~m} / \mathrm{s})$ depending on the movement model, demonstrating the suitability of the roaming procedure.

\section{Resource reusage}

Finally, we evaluate the capabilities of our TDMA approach for successful slot sharing and reuse between different cells. Since our design supports up to 1766 simultaneous users per superframe (see Section VI-A), slot collisions would almost never happen unless more than 1766 users are simulated, which is not possible in OMNeT++. Therefore, to evaluate the roaming process with slot collisions, we artificially limit the number of available slots to $n_{\text {slots }}=5$, while considering twice this number of mobile tags $n_{\text {tags }}=10$. During the remainder of the

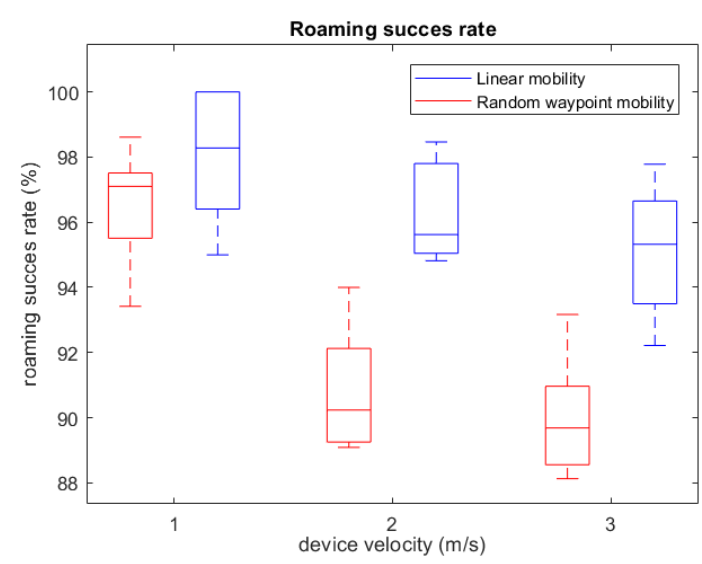

Fig. 5: Roaming success rate in function of tag velocity.

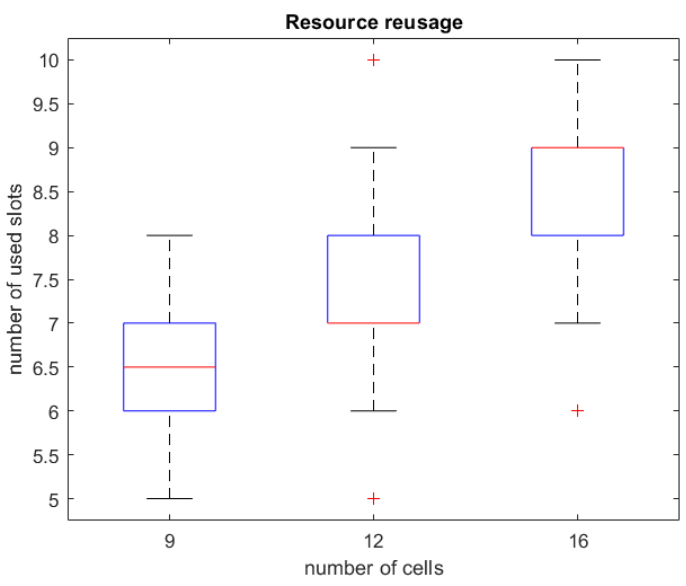

Fig. 6: Slot reuse in function of network size. Compared to the available number of slots $\left(n_{\text {slots }}=5\right.$ ), up to 10 slots can be assigned due to two-hop collision free slot sharing.

contention free period, the tags and anchor nodes remain in energy saving mode.

Three experiments were executed using networks with an increasing number of cells, ranging from 9 to 16 cells. In Figure 6, slot reusage is evaluated for the different network dimensions. The number of users that have received a slot is up to double the amount of available slots, e.g. for 16 cells up to 10 slots are successfully allocated (reused) while the superframe only defined 5 available slots. Slot reuse increases with increasing network size, as well as when users are spread more uniformly over the area. 


\section{CONCLUSIONS}

The need for accurate positioning in indoor environments has resulted in a significant amount of indoor localization research over the last years. In terms of localization accuracy, one of the best performing radio based technologies is UWB, which can provide cm-level accuracy. However, most of current research work focuses on realizing higher accuracies, rather than on supporting large scale multi-user and multi-cell deployments.

To remedy this, we proposed a new TDoA-TDMA multicell MAC protocol. The TDMA superframe consists of several parts, including (i) synchronization, (ii) slot allocation, (iii) slot reservation and (iv) dedicated ranging slots. For the synchronization of the anchor nodes and TDMA slots, UWB transmissions are used which are distributed efficiently through the network using the Trickle algorithm. For the slot allocation, we have proposed a scheduling algorithm that exploits the position estimates of the system and automatically calculates two-hop collision free regions based on the current and predicted sub-cell location of each tag.

The protocols have been implemented and evaluated using the OMNeT++ simulator, enhanced with probabilistic error models based on real-life measurements. The scalability was improved to $88.3 \%$ spectrum usage, resulting in 1766 slots per second, compared to only 720 slots for slotted ALOHA. Moreover, slots can be reused among multiple cells, and the roaming prediction success rate reaches over $90 \%$ accuracy, thereby demonstrating the feasibility of supporting multiple users in a large areas.

\section{ACKNOWLEDGMENT}

This work has partially been funded by the following projects: EC Interreg nano4sport, IMEC ICON LUNAR en Flanders Proeftuin Smart Connectivity

\section{REFERENCES}

[1] D. Dardari, P. Closas, and P. M. Djuric. "Indoor Tracking: Theory, Methods, and Technologies". In: IEEE Transactions on Vehicular Technology 64.4 (Apr. 2015), pp. 1263-1278.

[2] C. Zhang et al. "Real-Time Noncoherent UWB Positioning Radar With Millimeter Range Accuracy: Theory and Experiment". In: IEEE Transactions on Microwave Theory and Techniques 58.1 (Jan. 2010), pp. 9-20.

[3] Decawave. Decawave UWB ScenSor DW1000 Datasheet. URL: https://www.decawave.com/products/dw1000.

[4] Y. Qi et al. "Clear Channel Assessment (CCA) with multiplexed preamble symbols for impulse Ultra-wideband (UWB) communications". In: 2006 IEEE International Conference on Ultra-Wideband. Sept. 2006, pp. 675-680.

[5] N. Abramson. "THE ALOHA SYSTEM: Another Alternative for Computer Communications". In: Proceedings of the November 17-19, 1970, Fall Joint Computer Conference. AFIPS '70 (Fall). Houston, Texas: ACM, 1970, pp. 281-285.

[6] L. G. Roberts. "ALOHA Packet System with and Without Slots and Capture". In: SIGCOMM Comput. Commun. Rev. 5.2 (Apr. 1975), pp. 28-42.

[7] "IEEE Standard for Information Technology- Telecommunications and Information Exchange Between Systems- Local and Metropolitan Area Networks- Specific Requirements- Part 11: Wireless LAN Medium Access Control (MAC) and Physical Layer (PHY) Specifications". In: ANSI/IEEE Std 802.11, 1999 Edition (R2003) (2003), pp. i-513.

[8] S. Xu and T. Saadawi. "Does the IEEE 802.11 MAC protocol work well in multihop wireless ad hoc networks?" In: IEEE Communications Magazine 39.6 (June 2001), pp. 130-137.
[9] A. Ephremides and T. V. Truong. "Scheduling broadcasts in multihop radio networks". In: IEEE Transactions on Communications 38.4 (Apr. 1990), pp. 456-460.

[10] A. D. Gore and A. Karandikar. "Link Scheduling Algorithms for Wireless Mesh Networks". In: IEEE Communications Surveys Tutorials 13.2 (Feb. 2011), pp. 258-273.

[11] G. Wang and N. Ansari. "Optimal broadcast scheduling in packet radio networks using mean field annealing". In: IEEE Journal on Selected Areas in Communications 15.2 (Feb. 1997), pp. 250-260.

[12] C. Y. Ngo and V. O. K. Li. "Centralized broadcast scheduling in packet radio networks via genetic-fix algorithms". In: IEEE Transactions on Communications 51.9 (Sept. 2003), pp. 14391441.

[13] B. Radunovic and J. Y. L. Boudec. "Rate performance objectives of multihop wireless networks". In: IEEE Transactions on Mobile Computing 3.4 (Oct. 2004), pp. 334-349.

[14] L. van Hoesel and P. Havinga. "Collision-free Time Slot Reuse in Multi-hop Wireless Sensor Networks". In: 2005 International Conference on Intelligent Sensors, Sensor Networks and Information Processing. Dec. 2005, pp. 101-107.

[15] K. Amouris. "Position-based broadcast TDMA scheduling for mobile ad-hoc networks (MANETs) with advantaged nodes". In: MILCOM 2005 - 2005 IEEE Military Communications Conference. Oct. 2005, 252-257 Vol. 1.

[16] P. Sharma, S. Krishnan, and Z. Guoping. "A multi-cell UWBIR localization system for robot navigation". In: 2008 IEEE Radio and Wireless Symposium. Jan. 2008, pp. 851-854.

[17] G. E. Garcia et al. "Joint scheduling and localization in UWB networks". In: 2015 IEEE International Conference on Communication Workshop (ICCW). June 2015, pp. 724-729.

[18] M. Ridolfi et al. "WiFi ad-hoc mesh network and MAC protocol solution for UWB indoor localization systems". In: 2016 Symposium on Communications and Vehicular Technologies (SCVT). Nov. 2016, pp. 1-6.

[19] E. Karapistoli et al. "An overview of the IEEE 802.15.4a Standard". In: IEEE Communications Magazine 48.1 (Jan. 2010), pp. 47-53.

[20] "IEEE Standard for Local and metropolitan area networksPart 15.4: Low-Rate Wireless Personal Area Networks (LRWPANs)". In: IEEE Std 802.15.4-2011 (Revision of IEEE Std 802.15.4-2006) (Sept. 2011), pp. 1-314.

[21] Precision Time Protocol (PTP/IEEE-1588). Tech. rep. 2004.

[22] R. E. P. Loschmidt and G. Gaderer. "Highly Accurate Timestamping for Ethernet-Based Clock Synchronization". In: Journal of Computer Networks and Communications (2012).

[23] P. Levis et al. "Trickle: A Self-regulating Algorithm for Code Propagation and Maintenance in Wireless Sensor Networks". In: Proceedings of the 1st Conference on Symposium on Networked Systems Design and Implementation - Volume 1. NSDI'04. San Francisco, California: USENIX Association, 2004, pp. 2-2.

[24] OMNeT++ Discret Event Simulator. URL: https://omnetpp.org/.

[25] imec w-iLab.t Zwijnaarde - Generic Wireless Testbed. URL: http://wilab2.ilabt.iminds.be/.

[26] J. Tiemann, F. Eckermann, and C. Wietfeld. "Multi-user interference and wireless clock synchronization in TDOAbased UWB localization". In: 2016 International Conference on Indoor Positioning and Indoor Navigation (IPIN). Oct. 2016, pp. 1-6. 